\title{
Influence of rimonabant treatment on peripheral blood mononuclear cells; flow cytometry analysis and gene expression profiling
}

Stefan Almestrand, Xiao Wang, Åsa Jeppsson-Ahlberg, Marcus Nordgren, Jenny Flygare, Birger Christensson, Stephan Rössner, Birgitta Sander

The cannabinoid receptor type 1 (CB1) antagonist rimonabant has been used as treatment for obesity. In addition, anti-proliferative effects on mitogen-activated leukocytes have been demonstrated in vitro. We have previously shown that rimonabant (SR141716A) induces cell death in ex vivo isolated malignant lymphomas with high expression of CB1 receptors. Since CB1 targeting may be part of a future lymphoma therapy it was of interest to investigate possible effects on peripheral blood mononuclear cells (PBMC) in patients treated with rimonabant. We therefore evaluated leukocyte subsets by 6 color flow cytometry in eight patients before and at treatment with rimonabant for 4 weeks. Wholetranscript gene expression profiling in PBMC before and at 4 weeks of rimonabant treatment was done using Affymetrix Human Gene 1.0 ST Arrays. Our data show no significant changes of monocytes, B cells, total T cells or T cell subsets in PBMC during treatment with rimonabant. There was a small but significant increase in CD3-, CD16+ and/or CD56+ cells after rimonabant therapy. Gene expression analysis detected significant changes in expression of genes associated with innate immunity, cell death and metabolism. The present study shows that normal monocytes and leukocyte subsets in blood remain rather constant during rimonabant treatment. This is in contrast to the induction of cell death previously observed in CB1 expressing lymphoma cells in response to treatment with rimonabant in vitro. These differential effects observed on normal and malignant lymphoid cells warrant investigation of CB1 targeting as a potential lymphoma treatment. 


\section{Abstract}

5 The cannabinoid receptor type 1 (CB1) antagonist rimonabant has been used as treatment for

6 obesity. In addition, anti-proliferative effects on mitogen-activated leukocytes have been

7 demonstrated in vitro. We have previously shown that rimonabant (SR141716A) induces cell

8 death in ex vivo isolated malignant lymphomas with high expression of CB1 receptors. Since

9 CB1 targeting may be part of a future lymphoma therapy it was of interest to investigate possible

10 effects on peripheral blood mononuclear cells (PBMC) in patients treated with rimonabant. We

11 therefore evaluated leukocyte subsets by 6-color flow cytometry in eight patients before and at

12 treatment with rimonabant for 4 weeks. Gene expression profiling in PBMC before and at 4

13 weeks of rimonabant treatment was done using Affymetrix Human Gene 1.0 ST arrays. Our data

14 show no significant changes of B cells, total $\mathrm{T}$ cells or $\mathrm{T}$ cell subsets in PBMC during treatment with rimonabant. There was a small but significant increase in CD3-, CD16+ and/or CD56+ cells. Gene expression analysis detected significant changes in expression of genes associated with innate immunity, cell death and metabolism. The present study shows that leukocyte subsets in blood remain rather constant during rimonabant treatment. This is in contrast to the induction of cell death previously observed in CB1 expressing lymphoma cells in response to treatment with rimonabant in vitro. These differential effects observed on normal and malignant lymphoid cells warrant investigation of CB1 targeting as a potential lymphoma treatment. 
Stefan Almestrand ${ }^{1,2)}$, XiaoWang1,3), Åsa Jeppson-Ahlberg4), Marcus Nordgren ${ }^{1,5)}$,

25 Jenny Flygare ${ }^{1,6)}$, Birger Christensson ${ }^{1)}$, Stephan Rössner ${ }^{7)}$ and Birgitta Sander ${ }^{\text {*) }}$

27 1) Department of Laboratory Medicine, Division of Pathology, Karolinska Institutet and

Karolinska University Hospital Huddinge, Stockholm, Sweden

2) Present address: AstraZeneca, Södertälje, Sweden

3) Present address: Center for Primary Health Care Research, Skåne University Hospital,

Malmö, Sweden

4) Pathology/Cytology, Karolinska University Hospital Huddinge, Stockholm, Sweden

5) Present address: Laboratory of Lipid Biochemistry and Protein Interactions, Department of

6) Present address: Department of Laboratory Medicine, Division of Clinical Chemistry,

Karolinska Institutet, Stockholm Sweden

7) Department of Medicine, Karolinska University Hospital Huddinge, Stockholm, Sweden

*) Corresponding author

Corresponding author: Birgitta Sander, Dept. of Laboratory Medicine, Div. of Pathology, F46, Sweden.

Email: birgitta.sander@ki.se

Tel: +46858581044 Fax: +46858581020 


\section{Introduction}

The endocannabinoid system consists of the cannabinoid type 1 (CB1) and cannabinoid type 2 (CB2) receptors, their endogenous ligands anandamide and 2-arachidonoyl glycerol and the enzymes involved in their biosynthesis and metabolism (Di Marzo et al. 2004). CB1 is involved in the regulation of food intake, energy balance and metabolism of glucose and lipids (Di Marzo \& Matias 2005). In clinical studies CB1 receptor blockage by the selective CB1 antagonist rimonabant (SR141716A) induced weight loss and improvement in serum lipid, glucose and insulin levels by targeting central and peripheral CB1 receptors (Van Gaal et al. 2008). However, some patients experienced depressions and this was considered an unacceptable side effect for treating obesity/metabolic syndrome. Hence the drug was withdrawn from clinical use but there is remaining interest in some of its many potential medical applications (Cooper \& Regnell 2014; Zhou et al. 2012) including treatment of various malignancies. It is therefore of interest to investigate possible adverse effects on blood cells in patients treated with rimonabant.

The endocannabinoid system is regulating various aspects of lymphocyte proliferation, maturation and immune response (Klein 2005; Muppidi et al. 2011; Pandey et al. 2009; Pereira et al. 2009; Sido et al. 2014). Targeting the endocannabinoid system may therefore be a possible new treatment option in various lymphoproliferative disorders. CB1 receptors are expressed on cells of the immune system, but generally at lower levels than CB2 (Bouaboula et al. 1993; Galiegue et al. 1995). We and others have found that CB1 and CB2 are highly expressed on neoplastic lymphocytes in malignant lymphoma (Gustafsson et al. 2008; Islam et al. 2003; McKallip et al. 2002; Wasik et al. 2014). Targeting of CB1 and CB2 with endogenous or synthetic agonists reduced cell proliferation in vitro and in vivo and induced programmed cell 
death selectively in tumor cells of mantle cell lymphoma (Flygare et al. 2005; Gustafsson et al. 2006; Gustafsson et al. 2008; Wasik et al. 2011a). Similarly, CB2 agonists induced cell death in T cell lymphoblastic leukemia (McKallip et al. 2002). Also the CB1 antagonist rimonabant impaired proliferation and induced cell death in ex vivo isolated mantle cell lymphoma cells, alone, or in combination with anandamide (Flygare et al. 2005). Others have reported antiproliferative effects of rimonabant on in vitro activated PBMC but not on freshly isolated, non-activated PBMC (Gallotta et al. 2010; Malfitano et al. 2008). These results show that CB1 blockade may have immunomodulatory and antiproliferative effects on malignant lymphoma and on activated normal lymphocytes in vitro but seems to spare resting lymphocytes. Very little is published on effects of rimonabant on human PBMC in vivo and the aim of this study was to investigate how treatment with rimonabant affected blood leukocytes. We therefore collected blood cells from obese patients treated with rimonabant and analyzed blood leukocytes by flow cytometry before and during treatment. To investigate which genes were differentially expressed in PBMC during rimonabant treatment we used oligonucleotide arrays to compare gene expression profiles in PBMC before and at 4 weeks of treatment. This pilot study shows that rimonabant treatment induces expression of genes involved in immune responses but have only marginal effects on leukocyte subset frequencies in blood. 


\section{Materials and Methods}

Patients and study design:

Rimonabant was prescribed to eight patients, admitted to the Overweight Study Unit at the Department of Medicine, Karolinska University Hospital. Rimonabant was administered according to the manufacturers guidelines. All patients had a BMI $>35 \mathrm{~kg} / \mathrm{m}^{2}$, were treated on clinical indications (metabolic and mechanical disability) and without mental disturbances. They were not included in any other study. The clinical characteristics of these patients are presented in Table 1. Blood samples were collected before treatment and at the first clinical control, when the patients had received rimonabant, $20 \mathrm{mg}$ daily, for 4 weeks. All patients gave their informed consent and the study was performed in accordance with the Declaration of Helsinki and approved by the Regional Ethical Committee in Stockholm.

\section{Flow cytometry:}

The phenotypes of cells in the blood were analyzed by flow cytometry according to standard procedures at the Hematopathology Unit, Dept. of Pathology, Karolinska University Hospital, using 6 color flow cytometry to detect T, B, NK cells and CD3- CD4+ cells (monocytes and dendritic cells). Flow cytometry was performed on a CANTO 1 flow cytometer (BD, BectonDickinson, Europe).

For data acquisition and analysis, a CANTO 1 flow cytometer (BD, Becton Dickinson, Europe) was used with Cell Quest software (Becton Dickinson). All samples were analyzed by setting appropriate side and forward scatter gates to identify the mononuclear cell population, using CD45 and forward and side scatter for gate setting. Consistency of analysis parameters was ascertained by calibrating the flow cytometer with calibrating beads and FacsComp software, 
116 both from Becton Dickinson. The results are reported as percentage of gated cells positive for

117 each antibody. The following fluorochrome conjugated antibodies, all from BD, were used: CD4

118 PE, CD3 PerCP-Cy5.5, CD19 PE-Cy7, CD8 APC and CD45 APC-H7. We also used BD

119 Multitest 6-Color TBNK Reagent containing CD3 FITC clone SK7, CD16 PE clone B73, CD56

120 PE clone NCAM 16.2, CD45 PerCP-Cy5.5 clone 2D1, CD4 PE-Cy7 clone SK3, CD19 APC

121 clone SJ25C1 and CD8 APC-Cy7, clone SK1. The gating strategy is shown in Supplemental 122 Figure 1.

124 RNA isolation and oligonucleotide array hybridization:

125 Blood mononuclear cells were isolated by Ficoll separation (Ficoll-Paque PLUS, GE

126 Healthcare). From the cell-pellet total RNA was prepared using Qiagen midi plus kit (Qiagen

$127 \mathrm{GmbH}$, Hilden Germany) as recommended by the manufacturer and was quality controlled on an

128 Agilent Bioanalyzer (Agilent Technologies, Inc. Palo Alto, CA). Six pretreatment samples and

129 seven samples obtained after rimonabant treatment passed the quality control. The cRNA

130 synthesis for microarray experiments and the hybridizations were carried out using Affymetrix

131 Human Gene 1.0 ST Array (Affymetrix, Inc., Santa Clara, CA) according to standard Affymetrix

132 protocols at the core facility for Bioinformatics and Expression Analysis, Department of

133 Biosciences and Nutrition, Karolinska Institutet.

135 Gene expression data analysis:

136 We used tools provided in the Partek Genomic Suite 6.5 software (Partek Inc., St. Louis, MO).

137 Normalization was done by Robust Multiarray Analysis (RMA) followed by 1-way Analysis Of

138 Variance (ANOVA) comparing the patient group before and after treatment. Significantly 
139 changed genes and exons were selected with an unadjusted p-value of $<0,001$, a False

140 Discovery Rate (FDR) $<0,1$ and a fold-change equal or greater than $>1,5$ for up regulated genes

141 and equal or less than $<-1,5$ for down regulated genes. Gene functional annotations were

142 performed by using the free software DAVID v6.7 (Database for Annotation, Visualization and

143 Integrated Discovery) (Huang da et al. 2009). The gene expression data are deposited at the

144 GEO repository under the number GSE68055.

145

146

$147 \quad$ Statistical analysis

148 Leukocyte subsets (as measured by flow cytometry) in blood before and after rimonabant 149 treatment were analyzed using a paired t-test.

Results

Analysis of PBMC by flow cytometry before and during treatment with rimonabant

154 Blood levels of mononuclear cells on eight obese patients were analyzed by flow cytometry

155 before and during treatment with rimonabant. There were no significant changes in the relative

156 frequencies of total CD3+ T cells, CD4+ T cells, CD8+ T cells, B cells or CD3-CD4+ cells

157 (monocytes and dendritic cells) in the patients during the treatment period (Table 1, graphically

158 presented in Fig.1) There was however a trend towards an increase in percentage of CD3-,

$159 \mathrm{CD} 16+/$ and or CD56+cells (before treatment median 9\% range 7-14\%; after treatment median $16012 \%$ range $9-15 \% \mathrm{p}=0,049)$ (Table 1 , Fig. 1$)$. 
162

163

164

165

166

167

168

169

170

171

172

173

174

175

176

177

178

179

180

181

182

183 184

Whole-transcript gene expression analysis demonstrates significant changes in genes belonging

to innate immune system pathways.

Treatment with rimonabant might influence the expression of genes in patient leukocytes.

To explore possible differences in gene expression profiles, whole-transcript expression analysis of PBMC before and during treatment was done, using Affymetrix Human Gene 1.0 ST Arrays. 47 probe sets were significantly differently expressed during treatment with a fold change of at least $1,5,37$ probe sets showed increased expression and 10 decreased expression, respectively

(Table 1). Several of the genes with significantly increased expression after rimonabant treatment are known components of the innate immune system (as exemplified by KLRF1, LILRA2, CTSB, CD160, CD177, and LY96). KLRF1 (also named NKp80) encodes a lectin-type of receptor that is expressed on nearly all NK cells and stimulates their cytotoxicity and cytokine release (Kuttruff et al. 2009). LILRA2 is the gene for an immune receptor that is expressed on monocytes, B cells, NK cells and dendritic cells and affects antigen presentation and innate immune responses (Lu et al. 2012). CTSB encodes cathepsin B, a protein that can be expressed in several immune cells including monocytes and that is involved in cell migration and immune modulation (Staun-Ram \& Miller 2011). CD160 is an essential NK cell receptor and is involved in regulation of cytokine production (reviewed in (Le Bouteiller et al. 2011). CD177 is a GPI linked cell surface molecule that regulates activation and migration of neutrophil granulocytes (Stroncek 2007). LY96 (also named MD-2) is associating with toll-like receptor 4 and is involved in signaling by LPS (Mancek-Keber \& Jerala 2015). A few genes promoting increased apoptosis were also upregulated (BCL-like 13, an apoptosis facilitator (Jensen et al. 2014; Kataoka et al. 2001), RING1- and YY1-binding protein, a regulator of MDM2 (Chen et al. 2009)). 
185 It has previously been shown that chronic marijuana users have increased expression of CB1 in 186 peripheral blood mononuclear cells (Nong et al. 2002). We therefore specifically analyzed the 187 expression of genes belonging to the endocannabinoid system in our patient cohort. Rimonabant 188 treatment did neither affect the expression of CB1 (mean and standard deviation of CB1 expression values before and after treatment were 12,5 +/- 3,24 and 10,79+/- 2,43, respectively, corresponding to a fold change of $-1,1$ ) nor of CB2 or the enzymes involved in the degradation and/or synthesis of endocannabinoids (fatty acid amide hydrolase, FAAH, and N-acyl

phosphatidylethanolamine phospholipase D, NAPEPLD) either when analyzed by gene expression analysis or by RT-PCR (data not shown).

\section{Discussion}

In this study we investigated the possible effects on PBMC of treatment with the CB1 antagonist rimonabant in patients taking the drug for obesity. We found that the distribution of leukocyte subsets remained rather constant, as analyzed by flow cytometry before treatment and after 4 weeks of treatment with rimonabant with the exception of CD3-, CD16+ and/or CD56+ cells that increased after treatment. This subset includes NK cells (CD3-, CD56+ and/or CD16+) and also subsets of monocytes (CD3-CD16+). There were no significant changes in expression levels of cannabinoid receptors or enzymes involved in synthesis and metabolism of endocannabinoids. However gene expression analysis suggested that genes involved in metabolism, cell death and the innate immune system were up regulated during treatment. Rimonabant is the first selective CB1 antagonist registered for clinical use and was clinically developed for treatment of obesity and the metabolic syndrome. Beside the effect on food intake, anti-proliferative actions on normal and malignant cells have been reported. Cannabinoid 
receptors are often more highly expressed on malignant cells than on their normal counterparts and cancer cells are usually more sensitive to the action of cannabinoids than normal cells

210 (reviewed in (Flygare \& Sander 2007; Sido et al. 2014; Wasik et al. 2011b)). Rimonabant has

211 been reported to induce growth inhibition or apoptosis on several malignancies including breast, 212 thyroid and colon cancer (Bifulco et al. 2004; De Petrocellis et al. 1998; Santoro et al. 2009;

213 Sarnataro et al. 2006). We have previously demonstrated that mantle cell lymphoma and other B 214 cell lymphomas have higher expression of CB1 and CB2 than normal lymphocytes (Gustafsson 215 et al. 2008; Islam et al. 2003; Wasik et al. 2014). Cannabinoid receptor agonists, at 1-10 $\mu \mathrm{M}$ 216 levels, reduced proliferation and induced programmed cell death in mantle cell lymphoma in 217 vitro and in a xenotransplant model (Flygare et al. 2005; Gustafsson et al. 2006; Gustafsson et al. 2008; Schatz et al. 1997; Wasik et al. 2011a). Interestingly, similar concentrations of rimonabant induced cell death in ex vivo isolated mantle cell lymphoma cells (Flygare et al. 2005). While these studies suggest that targeting of CB1 may be of use in cancer therapy, concern may be raised since anti-proliferative effects have been reported in PBMC (Malfitano et al. 2008). In these studies rimonabant inhibited mitogen induced cell proliferation in vitro via G1/S phase arrest without induction of cell death (Malfitano et al. 2008). In contrast, Gallotta et al. reported that freshly isolated PBMC are highly resistant to the cytotoxic and cytostatic effects of rimonabant compared to leukemia-derived cell lines (Gallotta et al. 2010). Possibly, the different sensitivity to $\mathrm{CB} 1$ antagonism in freshly isolated, compared to mitogen activated, PBMC may reflect differences in expression levels of CB1. Resting leukocytes express very low levels of CB1 (Bouaboula et al. 1993; Galiegue et al. 1995; Kaminski et al. 1992) while receptor levels may increase upon activation by mitogens, cytokines or exposure to CB1 agonists (Borner et al. 230 2007; Nong et al. 2002; Schatz et al. 1997). We did not detect any significant differences in 
231 expression levels of CB1 or other components of the endocannabinoid system during rimonabant

232 treatment for 4 weeks. Furthermore, our studies on ex vivo isolated PBMC from rimonabant

233 treated patients could not demonstrated very minor changes in frequencies of $\mathrm{T}$ cells, B cells,

234 CD3-, CD16+ and/or CD56+ cells or CD3-CD4+ cells or on total lymphocyte counts, in line

235 with the results of Gallotta et al. (Gallotta et al. 2010).

236 Global gene expression analysis demonstrated significant changes in genes coding for

237 components of the innate immune system. The study design does not make it possible to

238 discriminate if the differences in gene expression can be ascribed to certain subsets of leukocytes

239 or if it is a general process, seen in all PBMC. However, many of the genes that were more

240 highly expressed after treatment with rimonabant are expressed in NK cells (such as KLRF1 and

$241 \mathrm{CD} 160)$ and monocytes, which imply that the treatment is associated with the activation of

242 certain inflammatory and immunological functions of the innate immune system. Interestingly,

243 rimonabant has been shown to directly activate human and mouse macrophages and thereby

244 inhibit the development of the intracellular pathogen Brucella suis (Gross et al. 2000).

245 Furthermore, studies on lipopolysaccharide activated human macrophages showed that CB1

246 receptor blockade by rimonabant suppressed production of inflammatory cytokines (IL-1 $\beta$, IL-6,

247 IL-8, TNF- $\alpha$ ) and matrix metalloproteinase-9 (Sugamura et al. 2009).

\section{Conclusions}

251 In conclusion our results show that rimonabant treatment induces expression of genes involved

252 in immune responses but have only marginal effects on leukocyte subset frequencies in blood.

253 This is in marked contrast to previous studies in which rimonabant induced cell death in 
malignant B lymphocytes that express high levels of CB1 (Flygare et al. 2005). The relatively

small effects on normal leukocytes suggest that CB1 targeting may be further investigated as a

therapeutic approach in lymphoma treatment, enabling selective effects of tumor cells.

\section{References}

Bifulco M, Laezza C, Valenti M, Ligresti A, Portella G, and V DIM. 2004. A new strategy to block tumor growth by inhibiting endocannabinoid inactivation. FASEB J 18:1606-1608.

Borner C, Hollt V, Sebald W, and Kraus J. 2007. Transcriptional regulation of the cannabinoid receptor type 1 gene in T cells by cannabinoids. J Leukoc Biol 81:336-343.

Bouaboula M, Rinaldi M, Carayon P, Carillon C, Delpech B, Shire D, Le Fur G, and Casellas P. 1993. Cannabinoid-receptor expression in human leukocytes. Eur J Biochem 214:173180.

Chen D, Zhang J, Li M, Rayburn ER, Wang H, and Zhang R. 2009. RYBP stabilizes p53 by modulating MDM2. EMBO Rep 10:166-172.

Cooper ME, and Regnell SE. 2014. The hepatic cannabinoid 1 receptor as a modulator of hepatic energy state and food intake. Br J Clin Pharmacol 77:21-30.

De Petrocellis L, Melck D, Palmisano A, Bisogno T, Laezza C, Bifulco M, and Di Marzo V. 1998. The endogenous cannabinoid anandamide inhibits human breast cancer cell proliferation. Proc Natl Acad Sci U S A 95:8375-8380.

Di Marzo V, Bifulco M, and De Petrocellis L. 2004. The endocannabinoid system and its therapeutic exploitation. Nat Rev Drug Discov 3:771-784.

Di Marzo V, and Matias I. 2005. Endocannabinoid control of food intake and energy balance. Nat Neurosci 8:585-589.

Flygare J, Gustafsson K, Kimby E, Christensson B, and Sander B. 2005. Cannabinoid receptor ligands mediate growth inhibition and cell death in mantle cell lymphoma. FEBS Lett 579:6885-6889.

Flygare J, and Sander B. 2007. The endocannabinoid system in cancer-Potential therapeutic target? Semin Cancer Biol.

Galiegue S, Mary S, Marchand J, Dussossoy D, Carriere D, Carayon P, Bouaboula M, Shire D, Le Fur G, and Casellas P. 1995. Expression of central and peripheral cannabinoid receptors in human immune tissues and leukocyte subpopulations. Eur J Biochem 232:54-61.

Gallotta D, Nigro P, Cotugno R, Gazzerro P, Bifulco M, and Belisario MA. 2010. Rimonabantinduced apoptosis in leukemia cell lines: activation of caspase-dependent and independent pathways. Biochemical pharmacology 80:370-380.

Gross A, Terraza A, Marchant J, Bouaboula M, Ouahrani-Bettache S, Liautard JP, Casellas P, and Dornand J. 2000. A beneficial aspect of a CB1 cannabinoid receptor antagonist: SR141716A is a potent inhibitor of macrophage infection by the intracellular pathogen Brucella suis. J Leukoc Biol 67:335-344.

Gustafsson K, Christensson B, Sander B, and Flygare J. 2006. Cannabinoid receptor-mediated apoptosis induced by $\mathrm{R}(+)$-methanandamide and Win55,212-2 is associated with 
ceramide accumulation and p38 activation in mantle cell lymphoma. Mol Pharmacol 70:1612-1620.

Gustafsson K, Wang X, Severa D, Eriksson M, Kimby E, Merup M, Christensson B, Flygare J, and Sander B. 2008. Expression of cannabinoid receptors type 1 and type 2 in nonHodgkin lymphoma: growth inhibition by receptor activation. Int J Cancer 123:10251033.

Huang da W, Sherman BT, and Lempicki RA. 2009. Systematic and integrative analysis of large gene lists using DAVID bioinformatics resources. Nat Protoc 4:44-57.

Islam TC, Asplund AC, Lindvall JM, Nygren L, Liden J, Kimby E, Christensson B, Smith CI, and Sander B. 2003. High level of cannabinoid receptor 1, absence of regulator of $G$ protein signalling 13 and differential expression of Cyclin D1 in mantle cell lymphoma. Leukemia 17:1880-1890.

Jensen SA, Calvert AE, Volpert G, Kouri FM, Hurley LA, Luciano JP, Wu Y, Chalastanis A, Futerman AH, and Stegh AH. 2014. Bc12L13 is a ceramide synthase inhibitor in glioblastoma. Proc Natl Acad Sci U S A 111:5682-5687.

Kaminski NE, Abood ME, Kessler FK, Martin BR, and Schatz AR. 1992. Identification of a functionally relevant cannabinoid receptor on mouse spleen cells that is involved in cannabinoid-mediated immune modulation. Mol Pharmacol 42:736-742.

Kataoka T, Holler N, Micheau O, Martinon F, Tinel A, Hofmann K, and Tschopp J. 2001. Bclrambo, a novel Bcl-2 homologue that induces apoptosis via its unique $\mathrm{C}$-terminal extension. J Biol Chem 276:19548-19554.

Klein TW. 2005. Cannabinoid-based drugs as anti-inflammatory therapeutics. Nat Rev Immunol 5:400-411.

Kuttruff S, Koch S, Kelp A, Pawelec G, Rammensee HG, and Steinle A. 2009. NKp80 defines and stimulates a reactive subset of CD8 T cells. Blood 113:358-369.

Le Bouteiller P, Tabiasco J, Polgar B, Kozma N, Giustiniani J, Siewiera J, Berrebi A, AguerreGirr M, Bensussan A, and Jabrane-Ferrat N. 2011. CD160: a unique activating NK cell receptor. Immunol Lett 138:93-96.

Lu HK, Mitchell A, Endoh Y, Hampartzoumian T, Huynh O, Borges L, Geczy C, Bryant K, and Tedla N. 2012. LILRA2 selectively modulates LPS-mediated cytokine production and inhibits phagocytosis by monocytes. PLoS One 7:e33478.

Malfitano AM, Laezza C, Pisanti S, Gazzerro P, and Bifulco M. 2008. Rimonabant (SR141716) exerts anti-proliferative and immunomodulatory effects in human peripheral blood mononuclear cells. Br J Pharmacol 153:1003-1010.

Mancek-Keber M, and Jerala R. 2015. Postulates for validating TLR4 agonists. Eur J Immunol 45:356-370.

McKallip RJ, Lombard C, Fisher M, Martin BR, Ryu S, Grant S, Nagarkatti PS, and Nagarkatti M. 2002. Targeting CB2 cannabinoid receptors as a novel therapy to treat malignant lymphoblastic disease. Blood 100:627-634.

Muppidi JR, Arnon TI, Bronevetsky Y, Veerapen N, Tanaka M, Besra GS, and Cyster JG. 2011. Cannabinoid receptor 2 positions and retains marginal zone B cells within the splenic marginal zone. J Exp Med 208:1941-1948.

Nong L, Newton C, Cheng Q, Friedman H, Roth MD, and Klein TW. 2002. Altered cannabinoid receptor mRNA expression in peripheral blood mononuclear cells from marijuana smokers. J Neuroimmunol 127:169-176. 
Pandey R, Mousawy K, Nagarkatti M, and Nagarkatti P. 2009. Endocannabinoids and immune regulation. Pharmacol Res 60:85-92.

Pereira JP, An J, Xu Y, Huang Y, and Cyster JG. 2009. Cannabinoid receptor 2 mediates the retention of immature B cells in bone marrow sinusoids. Nat Immunol 10:403-411.

Santoro A, Pisanti S, Grimaldi C, Izzo AA, Borrelli F, Proto MC, Malfitano AM, Gazzerro P, Laezza C, and Bifulco M. 2009. Rimonabant inhibits human colon cancer cell growth and reduces the formation of precancerous lesions in the mouse colon. Int J Cancer 125:9961003.

Sarnataro D, Pisanti S, Santoro A, Gazzerro P, Malfitano AM, Laezza C, and Bifulco M. 2006. The cannabinoid CB1 receptor antagonist rimonabant (SR141716) inhibits human breast cancer cell proliferation through a lipid raft-mediated mechanism. Mol Pharmacol 70:1298-1306.

Schatz AR, Lee M, Condie RB, Pulaski JT, and Kaminski NE. 1997. Cannabinoid receptors CB1 and CB2: a characterization of expression and adenylate cyclase modulation within the immune system. Toxicol Appl Pharmacol 142:278-287.

Sido JM, Nagarkatti PS, and Nagarkatti M. 2014. Role of Endocannabinoid Activation of Peripheral CB1 Receptors in the Regulation of Autoimmune Disease. Int Rev Immunol.

Staun-Ram E, and Miller A. 2011. Cathepsins (S and B) and their inhibitor Cystatin C in immune cells: modulation by interferon-beta and role played in cell migration. $J$ Neuroimmunol 232:200-206.

Stroncek DF. 2007. Neutrophil-specific antigen HNA-2a, NB1 glycoprotein, and CD177. Curr Opin Hematol 14:688-693.

Sugamura K, Sugiyama S, Nozaki T, Matsuzawa Y, Izumiya Y, Miyata K, Nakayama M, Kaikita K, Obata T, Takeya M, and Ogawa H. 2009. Activated endocannabinoid system in coronary artery disease and antiinflammatory effects of cannabinoid 1 receptor blockade on macrophages. Circulation 119:28-36.

Van Gaal L, Pi-Sunyer X, Despres JP, McCarthy C, and Scheen A. 2008. Efficacy and safety of rimonabant for improvement of multiple cardiometabolic risk factors in overweight/obese patients: pooled 1-year data from the Rimonabant in Obesity (RIO) program. Diabetes Care 31 Suppl 2:S229-240.

Wasik AM, Almestrand S, Wang X, Hultenby K, Dackland AL, Andersson P, Kimby E, Christensson B, and Sander B. 2011a. WIN55,212-2 induces cytoplasmic vacuolation in apoptosis-resistant MCL cells. Cell death \& disease 2:e225.

Wasik AM, Christensson B, and Sander B. 2011b. The role of cannabinoid receptors and the endocannabinoid system in mantle cell lymphoma and other non-Hodgkin lymphomas. Seminars in cancer biology 21:313-321.

Wasik AM, Nygren L, Almestrand S, Zong F, Flygare J, Baumgartner Wennerholm S, Saft L, Andersson P, Kimby E, Wahlin BE, Christensson B, and Sander B. 2014. Perturbations of the endocannabinoid system in mantle cell lymphoma: correlations to clinical and pathological features. Oncoscience s:550-557.

Zhou YH, Ma XQ, Wu C, Lu J, Zhang SS, Guo J, Wu SQ, Ye XF, Xu JF, and He J. 2012. Effect of anti-obesity drug on cardiovascular risk factors: a systematic review and meta-analysis of randomized controlled trials. PLoS One 7:e39062. 


\section{Legends to tables}

386

387

388

389

390

391

392

393

394

395

396

397

398

399

400

401

402

403

404

405

406

Table 1. Clinical parameters of included subjects and percentages of blood cells as analyzed by flow cytometry before and after 4 weeks of rimonabant treatment.

\# Values are percentage of cells in mononuclear gate. Total T cells were defined as CD3+, CD4+ T cells as $\mathrm{CD} 3+\mathrm{CD} 4+, \mathrm{CD} 8+\mathrm{T}$ cells as CD3+CD8+ and $\mathrm{B}$ cells as CD19+. The CD3-CD4+ cell population consists of monocytes and dendritic cells. The CD3-, CD16+ and/or CD56+ contains NK cells and also a subset of monocytes with CD16 expression. The CD3-, CD16+ and/or CD56+ cells increased after treatment ( $p=0,049$, paired t-test), all other changes were non significant.

Table 2. Genes differentially expressed in PBMC after treatment with rimonabant (ratio $>1,5$ between rimonabant treated and controls, $p$-value $<0,001$, false discovery rate $<0,1$ ).

\section{Figure legends}

Figure 1. Percentage of peripheral blood mononuclear cells (PBMC) before and during treatment with rimonabant. PBMC were analyzed by flow cytometry before start of therapy and 4 weeks later and results are given as percentage of mononuclear cells in blood. Each data point represents results from one patient. In cases with no change in the frequency of a certain cell type the data point would fall on the line. The only statistically significant change was for CD3-, CD16+ and/or CD56+ cells $(\mathrm{p}=0,049)$. For the other subsets the $p$-values were as follows: CD3+ $p=0,47 ; C D 3+C D 4+p=0,25 ; C D 3+C D 8+p=0,11 ; C D 19+p=0,13 ; C D 3-C D 4+p=0,11$. The 
407 mononuclear gate was defined by CD45 in combination with side and forward scatter. Within 408 this gate the frequencies of CD3+ T cells, CD19+ B cells, CD3-, CD56+ and/or CD16+ cells 409 (NK cells and subpopulation of CD16+ monocytes) and CD3-, CD4+ cells (monocytes and 410 dendritic cells) were analyzed.

411

412

413

414

415

416 


\section{Table $\mathbf{1}_{\text {(on next page) }}$}

Clinical parameters of included subjects and percentages of blood cells as analyzed by flow cytometry before and after 4 weeks of rimonabant treatment.

\# Values are percentage of cells in mononuclear gate. Total T cells were defined as CD3+, $C D 4+T$ cells as $C D 3+C D 4+, C D 8+T$ cells as $C D 3+C D 8+$ and $B$ cells as $C D 19+$. The CD3CD4+ cell population consists of monocytes and dendritic cells. The CD3-, CD16+ and/or CD56+ contain NK cells and monocytes with CD16 expression. There was a significant increase in CD3-, CD16+ and/Or CD56+ cells after treatment ( $p=0,049$, paired t-test), all other changes were non significant. 
3 Table 1. Clinical parameters of included subjects and percentages of blood cells as analyzed by flow cytometry before and after 44 weeks of rimonabant treatment.

\begin{tabular}{|c|c|c|c|c|c|c|c|c|c|c|c|c|c|c|}
\hline \multirow{3}{*}{ Patient } & \multirow{3}{*}{$\begin{array}{l}\text { Age, } \\
\text { sex }\end{array}$} & \multirow{3}{*}{$\begin{array}{c}\text { Weight } \\
\text { change } \\
\text { (kg) }\end{array}$} & \multicolumn{12}{|c|}{ Leukocyte subsets as analyzed by flow cytometry\# } \\
\hline & & & \multicolumn{2}{|c|}{ Total T cells } & \multicolumn{2}{|c|}{ CD4+ T cells } & \multicolumn{2}{|c|}{ CD8+ T cells } & \multicolumn{2}{|c|}{ B cells } & \multicolumn{2}{|c|}{ CD3-CD4+ cells } & \multicolumn{2}{|c|}{$\begin{array}{l}\text { CD3-, CD16+ } \\
\text { and/or CD56+ }\end{array}$} \\
\hline & & & before & after & before & after & before & after & before & after & before & after & before & after \\
\hline 1 & $50, \mathrm{~F}$ & nd & 75 & 73 & 61 & 59 & 14 & 13 & 15 & 12 & 7,2 & 8 & 9 & 14 \\
\hline 2 & $41, \mathrm{~F}$ & $-6,3$ & 58 & 61 & 37 & 34 & 21 & 26 & 32 & 25 & 7 & 6,6 & 8 & 12 \\
\hline 3 & $55, \mathrm{~F}$ & nd & 72 & 72 & 54 & 53 & 19 & 20 & 16 & 12 & 5,7 & 8 & 11 & 15 \\
\hline 4 & $56, \mathrm{M}$ & $-3,1$ & 70 & 72 & 48 & 51 & 21 & 20 & 15 & 17 & 5,9 & 4,6 & 14 & 10 \\
\hline 5 & $47, \mathrm{~F}$ & $-2,8$ & 75 & 73 & 51 & 49 & 23 & 24 & 11 & 12 & 4,9 & 8 & 13 & 15 \\
\hline 6 & $44, F$ & 0,0 & 69 & 70 & 51 & 50 & 17 & 19 & 20 & 18 & 3,9 & 4,6 & 8 & 12 \\
\hline 7 & $69, F$ & $-2,0$ & 81 & 81 & 37 & 35 & 45 & 46 & 8 & 8 & 3,3 & 3,4 & 9 & 11 \\
\hline 8 & $58, \mathrm{M}$ & $-3,0$ & 85 & 87 & 52 & 53 & 32 & 40 & 4 & 3 & 2,1 & 5,7 & 7 & 9 \\
\hline median & 52,5 & 2,9 & 73,5 & 72,5 & 51 & 50,5 & 21 & 22 & 15 & 12 & 5,3 & 6,15 & 9 & 12 \\
\hline (range) & (41-69) & $(0-6,3)$ & $(58-85)$ & $(61-87)$ & (37-61) & $(34-59)$ & $(14-45)$ & $(13-46)$ & $(4-32)$ & $(3-25)$ & $(2,1-7,2)$ & $(3,4-8)$ & $(8-14)$ & $(9-15)$ \\
\hline
\end{tabular}

9 
Table 2 (on next page)

Genes differentially expressed in PBMC after treatment with rimonabant (ratio $>1,5$ between rimonabant treated and controls, $p$-value $<0,001$, false discovery rate $<0,1$ ). 


\begin{tabular}{|c|c|c|c|c|}
\hline $\begin{array}{l}\text { Probe set } \\
\text { ID }\end{array}$ & Gene symbol & $\begin{array}{l}\text { Fold } \\
\text { change }\end{array}$ & p-value & Gene name \\
\hline 7953892 & KLRF1 & 2.50 & 0.00046 & $\begin{array}{l}\text { killer cell lectin-like receptor subfamily F, } \\
\text { member } 1\end{array}$ \\
\hline 7983910 & AQP9 & 2.25 & 0.00033 & aquaporin 9 \\
\hline 8031207 & LILRA2 & 2.10 & 0.00059 & $\begin{array}{l}\text { leukocyte immunoglobulin-like receptor, } \\
\text { subfamily A }\end{array}$ \\
\hline 8078008 & LSM3 & 2.00 & 0.00068 & LSM3 homolog \\
\hline 7981290 & WARS & 1.99 & 0.00028 & tryptophanyl-tRNA synthetase \\
\hline 8149330 & CTSB & 1.90 & 0.00089 & cathepsin B \\
\hline 8127534 & C6orf150 & 1.87 & 0.00035 & \\
\hline 7919243 & CD160 & 1.79 & 0.00096 & CD160 molecule \\
\hline 8130732 & BRP44L & 1.74 & 0.00073 & brain protein 44 -like \\
\hline 8110318 & PRELID1 & 1.72 & 0.00084 & PRELI domain containing 1 \\
\hline 8003953 & PSMB6 & 1.69 & 0.00013 & proteasome subunit, beta type, 6 \\
\hline 8015545 & RAB5C & 1.68 & 0.00085 & RAB5C, member RAS oncogene family \\
\hline 8133690 & MDH2 & 1.68 & 0.00081 & malate dehydrogenase 2 \\
\hline 8178676 & NEU1 & 1.67 & 0.00052 & sialidase 1 \\
\hline 7973110 & RNASE2 & 1.65 & 0.00028 & ribonuclease, RNase A family, 2 \\
\hline 026541 & FAM32A & 1.65 & 0.00032 & family with sequence similarity 32 , member $\mathrm{A}$ \\
\hline 8004247 & C17orf49 & 1.64 & 0.00062 & \\
\hline 8088820 & RYBP & 1.63 & 0.00076 & RING1 and YY1 binding protein \\
\hline 8058373 & WDR12 & 1.62 & 0.00077 & WD repeat domain 12 \\
\hline 8071119 & BCL2L13 & 1.61 & 0.00092 & BCL2-like 13 (apoptosis facilitator) \\
\hline 8174103 & GK & 1.61 & 0.00086 & glycerol kinase \\
\hline 8016099 & EFTUD2 & 1.60 & 0.00079 & $\begin{array}{l}\text { elongation factor Tu GTP binding domain } \\
\text { containing } 2\end{array}$ \\
\hline 7914563 & YARS & 1.60 & 0.00086 & tyrosyl-tRNA synthetase \\
\hline 979085 & PYGL & 1.59 & 0.00092 & phosphorylase, glycogen \\
\hline 8004237 & RNASEK & 1.59 & 0.00049 & ribonuclease, RNase K \\
\hline
\end{tabular}




\begin{tabular}{|l|l|l|l|l|}
\hline 7947681 & ARHGAP1 & 1.58 & 0.00025 & Rho GTPase activating protein 1 \\
\hline 7959153 & COX6A1 & 1.57 & 0.00096 & cytochrome c oxidase subunit VIa polypeptide 1 \\
\hline 8017437 & FTSJ3 & 1.56 & 0.00055 & FtsJ homolog 3 \\
\hline 8049180 & EIF4E2 & 1.55 & 0.00096 & $\begin{array}{l}\text { eukaryotic translation initiation factor 4E family } \\
\text { member }\end{array}$ \\
\hline 8146934 & LY96 & 1.54 & 0.00075 & lymphocyte antigen 96 \\
\hline 7900922 & ATP6V0B & 1.54 & 0.00064 & ATPase, H+ transporting \\
\hline 8037913 & NAPA & 1.53 & 0.00037 & $\begin{array}{l}\text { N-ethylmaleimide-sensitive factor attachment } \\
\text { protein, alpha }\end{array}$ \\
\hline 8037037 & ATP5SL & 1.52 & 0.00031 & ATP5S-like \\
\hline 8016708 & LRRC59 & 1.51 & 0.00019 & leucine rich repeat containing 59 \\
\hline 8163383 & SUSD1 & 1.51 & 0.00062 & sushi domain containing 1 \\
\hline 7990151 & PKM2 & 1.51 & 0.00093 & pyruvate kinase, muscle \\
\hline 7978123 & PSME2 & 1.51 & 0.00065 & proteasome activator subunit 2 \\
\hline 8075564 & RFPL2 & -1.51 & 0.00020 & ret finger protein-like 2 \\
\hline 7900878 & ARTN & -1.51 & 0.00041 & artemin \\
\hline 8141228 & TMEM130 & -1.51 & 0.00075 & transmembrane protein 130 \\
\hline 8037298 & CD177 & -1.58 & $4.0 \mathrm{e}-005$ & CD177 molecule \\
\hline 8069142 & KRTAP10-4 & -1.59 & 0.00023 & keratin associated protein 10-4 \\
\hline 8070771 & KRTAP10-1 & -1.60 & 0.00054 & keratin associated protein 10-1 \\
\hline 8172713 & LOC347549 & -1.61 & 0.00061 & hypothetical LOC347549 \\
\hline 8075200 & RHBDD3 & -1.63 & 0.00067 & rhomboid domain containing 3 \\
\hline 8167575 & GAGE12B & -1.66 & 0.00028 & G antigen 12B \\
\hline 8010901 & DOC2B & -1.82 & 0.00072 & double C2-like domains, beta \\
\hline
\end{tabular}




\section{Figure 1 (on next page)}

Percentage of peripheral blood mononuclear cells (PBMC) before and during treatment with rimonabant.

PBMC were analyzed by flow cytometry before start of therapy and 4 weeks later and results are given as percentage of mononuclear cells in blood. Each data point represents results from one patient. In cases with no change in the frequency of a certain cell type the data point would fall on the line. The only statistically significant change was for CD3-, CD16+ and/or CD56+ cells $(p=0,049)$. For the other subsets the $p$-values were as follows: CD3+ $p=0,47 ; C D 3+C D 4+p=0,25 ; C D 3+C D 8+p=0,11 ; C D 19+p=0,13 ; C D 3-C D 4+p=0,11$. The mononuclear gate was defined by CD45 in combination with side and forward scatter. Within this gate the frequencies of CD3+ T cells, CD19+ B cells, CD3-, CD56+ and/or CD16+ cells (NK cells and subpopulation of CD16+ monocytes) and CD3-, CD4+ cells (monocytes and dendritic cells) were analyzed. 

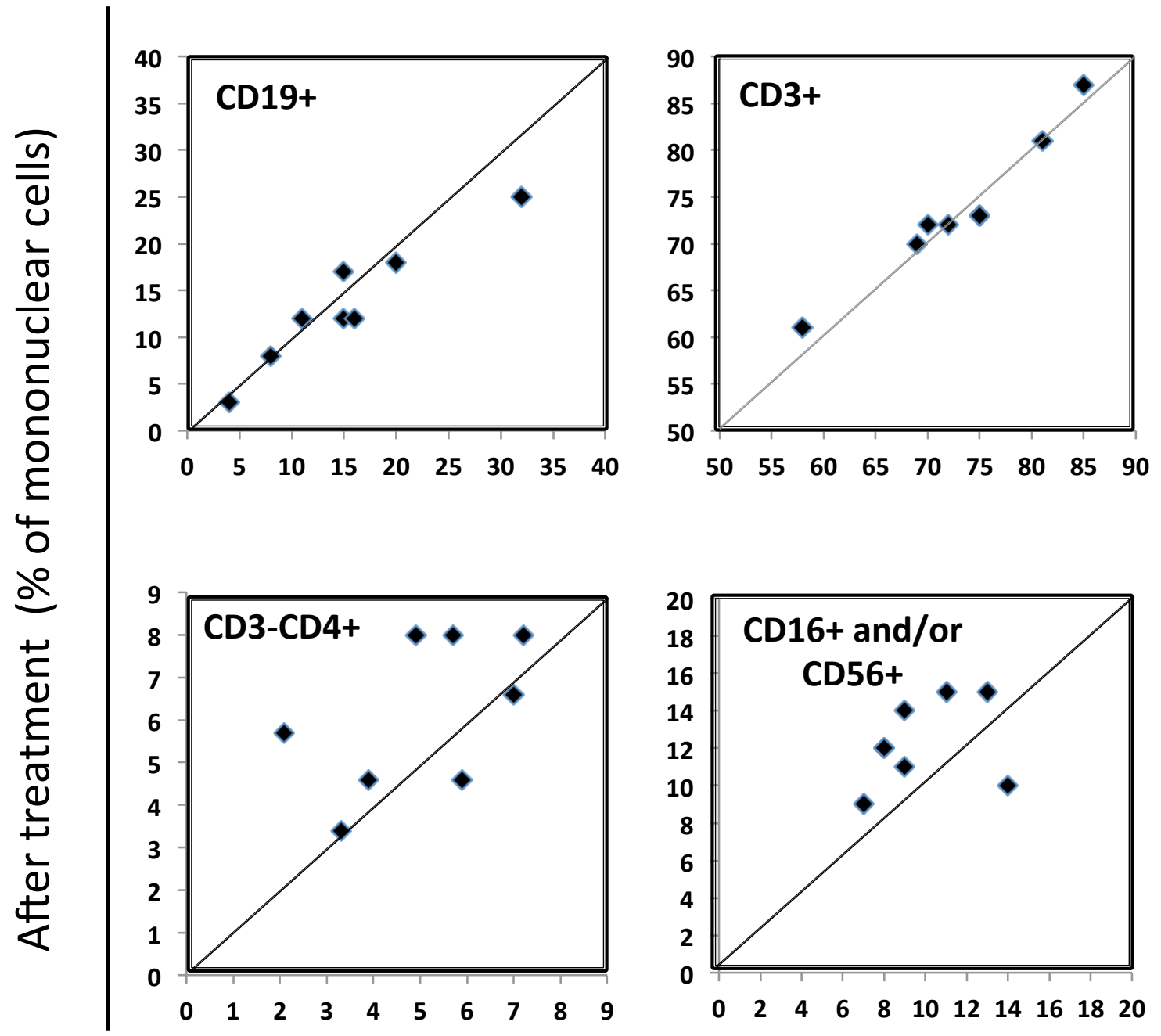

Before treatment (\% of mononuclear cells) 